BULL. AUSTRAL. MATH. SOC.

VOL. 16 (1977), 479-480.

\title{
Variability of Room squares
}

\section{Ian Robert Beaman}

An algorithm for the generation of the equivalence classes of Room squares of side nine is developed, along with a search for skew Room squares of side nine. Some ancillary results concerning incomplete Room squares are given, and some questions on the existence of skew Room squares in general are considered.

Chapter 1 is concerned with introducing the relevant theory on Room squares; their existence, isomorphism, the existence of isomorphism invariants, their relationship to one-factorizations and Latin squares, and their construction from starters. It also gives a summary of the relevant elements of graph theory and Steiner triple systems.

In Chapter 2 some results for incomplete Room squares are developed. In particular, the incidence graph of an incomplete Room square is characterised, and the starter method for construction is extended to apply to incomplete Room squares and the resulting squares are examined graphically.

Chapter 3 is devoted to identifying the equivalence classes of Room squares of side 9 . Room squares of side 9 are constructed using the starter method and orthogonal Steiner systems of side nine are shown to be non-existent. An algorithm for determining the inequivalent Room squares of side nine is detailed. Room squares are constructed from mutually orthogonal factorizations starting with the 396 inequivalent factorizations of $K_{10}$. Those Room squares found to be isomorphic to another, or to the transpose of another are also identified, and the remaining are listed in an Appendix as representing the inequivalent Room

Received 19 April 1977. Thesis submitted to the University of Newcastle, New South Wales, February 1977. Degree approved, April 1977. Supervisor: Associate Professor W.D. Wallis. 
squares of side 9 . A skew Room square of side 9 is found, and from generalisations of existence theorems for Room squares made to apply to skew Room squares, the question of existence of skew Room squares in general is condensed to a relatively small number of cases.

Chapter 4 gives the origin of the data used in the algorithm outlined in Chapter 3, and gives the automorphism groups of the Room squares constructed in that Chapter. The computer programs based on the algorithm are also described in detail. 